\section{Questión}

Periodismo / Comunicación $155 \mathrm{~N} 1669.6581$

Políticas de comunicación en el campo académico. Algunas precisiones teórico metodológicas Serguei Komissarov

Question/Cuestión, Nro.70, Vol.3, diciembre 2021

ISSN: $1669-6581$

URL de la Revista: https://perio.unlp.edu.ar/ojs/index.php/question/

IICom -FPyCS -UNLP

DOI: https//doi.org/10.24215/16696581e636

\title{
Políticas de comunicación en el campo académico
}

Algunas precisiones teórico metodológicas

\section{Communication policies in the academic field}

\section{Some theoretical and methodological details}

Serguei Komissarov

Facultad de Periodismo y Comunicación Social. Universidad Nacional de La Plata

Argentina

skomissarov@protonmail.com

https://orcid.org/0000-0002-7785-8369

\section{Resumen}

El presente artículo es fruto de un trabajo cuyo objetivo fue analizar la incorporación de la Ley de Servicios de Comunicación Audiovisual (LSCA) como objeto de estudio en la agenda de investigación del campo académico de la comunicación entre la sanción de la normativa y su plena vigencia (2009-2013) para dar cuenta de la articulación entre la política pública relacionada con los medios masivos de comunicación y las prácticas académicas, 
institucionales y políticas de los agentes del ámbito universitario. Este artículo pretende aportar a la construcción de un marco teórico metodológico para delimitar el objeto de estudio y problematizar la relación entre la transformación en las políticas públicas de comunicación y la producción académica al respecto.

Palabras claves: Ley de Servicios de Comunicación Audiovisual - campo académico - marco teórico metodológico - políticas de comunicación

\section{Abstract}

This article is the result of a work whose objective was to analyze the incorporation of the Audiovisual Communication Services Law (LSCA) as an object of study in the research agenda of the academic field of communication between the sanction of the regulation and its full validity (2009-2013) to account for the articulation between public policy related to the mass media and the academic, institutional and political practices of university agents. This article aims to contribute to the construction of a theoretical methodological framework to define the object of study and to problematize the relationship between the transformation in public communication policies and academic production in this regard.

Key words: Audiovisual Communication Services Law; academic field; methodological theoretical framework; communication policies

\section{Introducción}

La pregunta que guía el presente trabajo intenta dar cuenta de la articulación entre la política pública relacionada con los medios masivos de comunicación y las prácticas académicas, institucionales y políticas de los agentes del ámbito universitario. El objetivo general consiste en analizar y describir la incorporación de la Ley de Servicios de Comunicación Audiovisual como objeto de estudio en la agenda de investigación del campo académico de la comunicación en el período 2009-2013.

Para emprender esta tarea segmentamos nuestro objetivo general en varios específicos. Estos son: 
Relevar y analizar los artículos publicados en las revistas argentinas especializadas en comunicación en torno a la LSCA durante el período 2009-2013.

Relevar y analizar las ponencias de los congresos anuales de la Red de Carreras de Comunicación Social y Periodismo de Argentina (RedCom) referidas a la LSCA durante el período 2009-2013.

Relevar los testimonios de los principales referentes de la disciplina del país en torno a la incorporación de la LSCA en los estudios de comunicación.

Analizar los posicionamientos político-intelectuales de los referentes del campo sobre la LSCA.

\section{Algunas precisiones conceptuales}

A propósito de la propuesta general, es necesario dar cuenta de una serie de concepciones teóricas para comprender de qué manera la LSCA se incorpora como objeto de estudio en la agenda de investigación del campo académico de la comunicación.

En primer término, resulta relevante estudiar cuáles son las relaciones que se establecen entre el Estado y los diversos actores sociales en la implementación de políticas de comunicación desde una concepción, tanto teórica como metodológicamente, capaz de analizar las diferentes dimensiones, vínculos e interacciones que supone la formulación de políticas públicas en el marco de regímenes democráticos.

\section{Políticas públicas de comunicación}

En principio, se hace necesario definir la noción de políticas públicas. Para ello se recurrirá a los estudios de Oszlak y O'Donnell sobre la aplicación de políticas estatales en América Latina, que los autores definen como «un conjunto de acciones y omisiones que 
manifiestan una determinada modalidad de intervención del Estado en relación con una cuestión que concita la atención, interés o movilización de otros actores de la sociedad civil» (Oszlak y O'Donnell, 1984:10). Esta definición nos permite entender que la decisión política de un gobierno para actuar o no sobre determinado problema puede conformar en sí una política pública.

En este mismo sentido, Kauffer explica que una política pública no es necesariamente una acción concreta, sino que también puede implicar una acción simbólica o un programa ya que «las políticas públicas se refieren a actividades materiales o simbólicas que gestionan las autoridades públicas» (Kauffer, 2002:3). Por otra parte, la autora puntualiza un elemento clave de la definición referido a las políticas públicas, entendidas como el conjunto de decisiones cuyo objeto es la distribución de determinados bienes. Esto enfatiza el hecho de que, en las tomas de decisión, invariablemente se encuentran en juego recursos que pueden ser distribuidos para favorecer o afectar a determinados grupos sociales por sobre otros.

Ahora bien, como vimos anteriormente, en la definición de política pública subyace implícitamente el rol del Estado, al cual, se hace necesario conceptualizar teóricamente. En este caso, tomaremos la propuesta de O'Donnell, quien sostiene que el Estado:

Es una asociación con base territorial, compuesta de conjuntos de instituciones y de relaciones sociales (la mayor parte de ellas sancionadas y respaldadas por el sistema legal de ese Estado) que normalmente penetra y controla el territorio y los habitantes que ese conjunto delimita. Esas instituciones reclaman el monopolio en la utilización legítima del uso de la coerción física y normalmente tienen, como último recurso para efectivizar las decisiones que toman, supremacía en el control de los medios de esa coerción sobre la población y el territorio que el Estado delimita (O'Donnell, 2010:76).

Esta definición posee varios supuestos que es conveniente aclarar. En primer lugar, el Estado se compone de una serie de instituciones burocráticas de organización compleja que tienen por responsabilidad la protección o logro de algún aspecto del bien común actuando 
para efectivizar derechos, libertades y obligaciones correspondientes a cada ciudadano. Por supuesto que esto supone la existencia de un conjunto de reglas legales que sirven para sancionar y respaldar los derechos y las libertades de los habitantes de dicho territorio. Además, la definición aclara que las instituciones estatales «reclaman el monopolio en la utilización legítima del uso de la coerción física». Esta concepción entiende que el Estado reclama el monopolio de la autorización legítima del uso de la coerción física y, sólo como consecuencia de ello, reclama el monopolio del uso legítimo de dicha fuerza, cuya legitimación se produce a partir de normas legales (O'Donnell, 2010).

Una vez definido el rol de Estado, cabe preguntarnos cuál es la especificidad de las políticas públicas relacionadas con los medios masivos de comunicación. Para intentar definirlas utilizaremos la conceptualización de las políticas culturales y de comunicación que realiza Bustamante, entendidas como:

Las acciones y las omisiones de las instancias estatales de todo tipo que, de acuerdo con las concepciones y legitimaciones de cada sociedad y cada tiempo histórico, determinan u orientan los destinos de la creación, la producción, difusión y consumo de los productos culturales y comunicativos (Bustamante, 2004:10).

Por su parte, Sierra afirma que la noción de políticas de comunicación incluye «la definición de objetivos, disposiciones y actividades de las instituciones y de los actores políticos conducentes a organizar y lograr desarrollos específicos con relación a las condiciones de materialización de la comunicación pública moderna (prensa, radio, cine, televisión, nuevas tecnologías, etc.) » (Sierra, 2006:25).

En este sentido, podemos concluir en una definición operativa de políticas de comunicación sintetizando los aportes teóricos de distintos autores. De esta forma, entendemos por tales al conjunto de acciones $u$ omisiones que representan determinada forma de intervención estatal (Oszlak y O'Donnell, 1984) en materia de comunicación, a fin de elaborar una serie de normas que regulen el funcionamiento de los medios de difusión masiva tanto públicos como privados- en un Estado determinado (Graziano, 1988). 
Una vez definidas las políticas públicas de comunicación, pasemos a conceptualizar la relación entre éstas y el conjunto de la sociedad civil. Para examinar la gestión estatal y sus consecuencias en la sociedad se implementará la perspectiva relacional desarrollada por Bob Jessop (1982). Este enfoque posibilita alejarse, por un lado, de aproximaciones sociocéntricas que explican la acción del estado sobre la base de los intereses, las relaciones y estructuras de la sociedad civil y, por el otro, de la perspectiva que coloca el énfasis en la burocracia estatal y su forma de plantear un determinado ordenamiento. Por el contrario, tal como afirma Kriger:

Jessop propone una perspectiva relacional y coyuntural que permite problematizar los efectos de la intervención del Estado, examinando tanto las mediaciones institucionales como los sectores sociales involucrados. En el juego de relaciones, se observan claramente las capacidades de los diferentes actores para alcanzar sus objetivos a través del conflicto y la negociación; por ese motivo, según esta perspectiva, la distribución inicial de recursos de poder no predetermina las respuestas que el Estado pone en marcha frente a la movilización de los actores sociales, ni las formas en que estos procesos se desenvuelven (Kriger, 2009:19).

Siguiendo a Jessop, podemos afirmar que la LSCA no fue una iniciativa impuesta mecánicamente desde arriba por el poder político, pero tampoco, solamente el fruto de una lucha histórica mantenida por diversos actores sociales en contra de la concentración mediática. Por el contrario, la normativa responde, en todo caso, a la voluntad política de un gobierno enfrentado a corporaciones mediáticas que fue capaz de hacerse eco de las demandas de varios grupos y colectivos de la sociedad civil y lograr el consenso necesario para convertirlas en una normativa destinada a reordenar el mapa mediático en el país.

\section{Medios de comunicación y democracia}

Todo sistema democrático supone la existencia de tres elementos esenciales: elecciones limpias, decisivas e institucionalizadas como mecanismo para alcanzar los cargos del Estado; la presencia de derechos positivos y participativos de votar y ser elegidos; y las libertades necesarias para que existan ese tipo de elecciones: libertad de expresión, de asociación y de información (O’Donnell, 2010). 
En este contexto, la libre circulación de información e ideas resulta preponderante a fin de que los ciudadanos sean capaces de conocer, discutir y decidir sobre aquellas cuestiones de interés general que involucran al conjunto de la sociedad. En este sentido, puede afirmarse que no existen sociedades realmente democráticas sin una comunicación democrática. Esto equivale a sostener que no puede existir un sistema político genuinamente democrático sin medios de comunicación que den cuenta de la diversidad política y cultural de la sociedad.

Tal como explica Roncagliolo, «los sistemas de comunicación se definen con relación a la democracia» (1983: 51). En caso de que prevalezca una «des-semantización» del concepto de democracia, entendida no en términos políticos sino como una «democracia del consumo» a modo de una ilusión de consumo igualitario-, el sistema de comunicación se definiría, por su parte, por la ilusión de una participación informativa. Los atributos del ciudadano, se convierten así, en los del receptor/consumidor: ambos poseen «libertad de» (elección y consumo) en lugar de «libertad para». Se promueve, de esta forma, una «libertad-autonomía» atomizante en vez de una «libertad-participación» integradora.

Es precisamente por ello que la democratización de las comunicaciones adquiere suma relevancia ya que, sólo con medios de comunicación democráticos de información confiable y diversa, lo ciudadanos son capaces de debatir, proponer y actuar en el espacio público donde se dirimen las cuestiones políticas. Por eso, tal como señala Perry Anderson:

Una democracia profunda precisa de un sistema parlamentario fuerte, basado en partidos disciplinados, con financiamiento público equitativo $y$, sobre todo, exige una democratización de los medios de comunicación, cuyo monopolio en manos de ciertos grupos capitalistas súper-concentrados y prepotentes es incompatible con cualquier justicia electoral o soberanía democrática real (Anderson, 1999:147).

Llegados a este punto no podemos obviar en nuestro análisis la influencia del poder económico en la relación entre comunicación y democracia. Si bien no es el objetivo principal 
de este trabajo, consideramos necesario realizar un breve recorrido histórico que nos permita comprender el estado actual de la cuestión relativa a la concentración mediática (1).

Uno de los primeros antecedentes sobre los riesgos de la concentración mediática data de los años '60. La llamada teoría de la dependencia surge en América Latina al calor de numerosos procesos de cambio social ocurridos en todo el mundo. Por mencionar tan sólo algunos de los ejemplos de este fenómeno pueden citarse la revuelta obrero estudiantil de París en 1968 -conocida luego como el Mayo Francés-, la culminación del proceso de descolonización en África, la renovación de la Iglesia Católica a través del Concilio Vaticano II junto con el surgimiento del movimiento de la teología de la liberación que buscaba reconciliar la Institución con los pobres y la definitiva consolidación de la Revolución Cubana luego del fracasado golpe militar orquestado por Estados Unidos en Playa Girón.

Como respuesta a esta inusitada efervescencia social la administración de John F. Kennedy comienza a implementar una serie de políticas para promover el desarrollo de un capitalismo reformista en América Latina mediante un programa conocido como Alianza Para el Progreso. El plan consistía, básicamente, en una ayuda económica de inversiones estadounidenses a través de agencias financieras multilaterales con el supuesto objetivo de mejorar las condiciones sanitarias, ampliar acceso a la educación y la vivienda e incrementar la productividad agrícola mediante una reforma agraria y la implementación de nuevas tecnologías. De esta manera, se esperaba frenar el avance comunista en el continente en el polarizado contexto de la Guerra Fría.

Por otra parte, ya a mediados de los '60, comenzó a notarse el fracaso de las teorías propuestas por la CEPAL, dirigida entones por Raúl Prebisich, que planteaba la necesidad de un desarrollo dirigido por un Estado con capacidad para intervenir eficazmente en la esfera económica. De esta forma, se creía, América Latina reunía todas las condiciones para lograr un crecimiento autosostenido. Sin embargo, al cabo de muchos años de esta prédica y de la aplicación de las políticas económicas inspiradas por la CEPAL, la región persistía en su atraso.

Esta situación llevó a repensar la teoría clásica sobre el imperialismo en un nuevo contexto en el cual los países de América Latina habían dejado de ser colonias para 
convertirse en estados-nación formalmente independientes. En 1964 André Gunder Frank, un alemán especialista en historia económica latinoamericana, publica el libro Capitalismo y subdesarrollo en América Latina en el que sostiene que esta región del mundo es atrasada y subdesarrollada no a causa de la ausencia de estructuras y procesos capitalistas sino, precisamente, debido a ellos. De este modo, para Gunder Frank:

El capitalismo es una especie de sistema solar, que tiene un centro: las metrópolis imperiales, y una serie de satélites que giran, subordinados, a su alrededor. Pero ese giro no es permanente sino cíclico; durante un tiempo esas economías satelizadas llegan a experimentar un gran crecimiento de sus exportaciones. Se trata de un desarrollo parcial, altamente desequilibrado y completamente dependiente de la demanda metropolitana; una vez concluida esa fase esas economías se sumergen en un proceso de progresivo subdesarrollo. Entonces, concluye Frank, el causante del subdesarrollo de América Latina es el tipo de desarrollo capitalista que exige su inserción en el mercado mundial. Este no hace otra cosa que generar un ciclo de expansión, de crecimiento de las exportaciones, en detrimento del mercado interno y de un genuino desarrollo de las fuerzas productivas. Cuando este período entra en crisis el país queda peor que antes (Borón, 2008).

La crítica se centraba en los términos de intercambio comercial entre Estados Unidos y Latinoamérica según los cuales los países de la región eran obligados a vender materias primas a bajo costo y comprar productos manufacturados a precios elevados causando un permanente déficit, agravado, además, por las altas tasas de interés de los préstamos estadounidenses que endeudaban continuamente la periferia.

En esta compleja trama de dependencia y, gracias los primeros estudios en comunicación(2), comienza a formarse la idea de que la dominación no era solamente de orden político y económico, sino que, además, incluía una dimensión cultural. Este tipo de dominación se entendió como: 
La imposición de valores, creencias, normas y comportamientos de la minoría gobernante sobre la mayoría desvalida y oprimida. Se anotó que ella se logra mediante los procesos de socialización y control social que se dan tradicionalmente por conducto de la educación - en la escuela y en el hogar - y del adoctrinamiento religioso, así como contemporáneamente, por recurso a la comunicación masiva vía prensa, radio, televisión y cine. Y se afirmó que la finalidad de la dominación cultural es la de persuadir al pueblo raso de que piense, sienta y actúe de manera que contribuya con ignorancia, desconcierto, pasividad y conformidad a la conservación del régimen oligárquico que lo subordina, damnifica y silencia (Beltrán, 2007).

Por supuesto que la dominación cultural estaba estrechamente relacionada con la económica, por lo cual, además de discutir la función ideológica de los medios de comunicación comenzó a denunciarse su carácter monopólico. La mayoría de las agencias publicitarias y de noticias, las encuestadoras de opinión pública, más de la mitad de las películas cinematográficas y los contenidos radiales y televisivos, así como las publicaciones impresas provenían directamente de Estados Unidos o eran provistas por empresas nacionales asociadas con capitales extranjeros.

Tal es así que el Movimiento de Países No Alineados, comenzó a denunciar este monopolio informativo a escala mundial. Este diagnóstico culminó con la presentación del famoso Informe McBride en la XXI Conferencia General de la UNESCO en 1980. Además de un detallado estado de la situación acerca de la concentración informativa, el texto contenía una serie de conceptos sobre los cuales debía basarse un Nuevo Orden Mundial de la Información y la Comunicación (NOMIC)(3). Entre ellos, se sostenía la necesidad de suprimir los monopolios mediáticos y propiciar la pluralidad de fuentes y canales informativos.

Sin embargo -en la década del ochenta-, luego del triunfo mundial de las ideas neoliberales, las propuestas del Informe McBride fueron condenadas al fracaso. Debido a que la iniciativa descansaba sobre la base de la cooperación y solidaridad internacional, la falta de apoyo por parte de las potencias desarrolladas, afectadas en sus intereses económicos, truncó definitivamente la posibilidad del acceso a las nuevas tecnologías y a la libre circulación de información. 
A partir de aquel momento, la etapa neoliberal se caracterizó por el abandono de las tesis que sostenían la necesidad de una fuerte presencia estatal en la esfera económica, incluyendo por supuesto, las políticas relacionadas con los medios de comunicación. El triunfo de las teorías que postulaban la supuesta autorregulación del mercado posibilitó la implementación de una serie de reformas que agudizaron una estructura de propiedad dominada por el capital concentrado. Mediante la legitimación del discurso de libre mercado, grandes sectores de origen industrial y financiero comenzaron a invertir en el mercado comunicacional propiciando un fuerte proceso de concentración. Por supuesto que para ello se debieron adaptar los mecanismos legales con el objeto de hacerlos compatibles con los nuevos intereses económicos.

En el caso argentino, durante la década de los '90, se asistió a un fuerte proceso de privatizaciones de canales televisivos, emisoras radiales y empresas de telefonía pública que posibilitó una reestructuración del mercado de medios de comunicación y acentuó la concentración de la propiedad. Estos cambios fueron propiciados por modificaciones legales(4) que originaron los multimedios nacionales existentes en la actualidad. (5)

Ahora bien, ¿cuáles son las consecuencias de este proceso de concentración? Siguiendo a Becerra y Mastrini (2006), podemos agrupar los efectos de la concentración en tres categorías:

1. La tendencia a la unificación de las líneas editoriales producto de la reducción de voces.

2. La existencia de conflicto de intereses entre el propósito informativo y el entramado económico del grupo de comunicación concentrado, lo que lleva a que la información política y económica (tanto la que se brinda como la que se omite) esté filtrada por estos intereses.

3. La necesidad de concretar economías de escala y reducir costos laborales y administrativos, cuya consecuencia es un aprovechamiento máximo de los recursos físicos y humanos con la consecuente merma en la calidad de los contenidos. 
A modo de conclusión de lo expuesto anteriormente, podemos afirmar que el pluralismo y la multiplicidad de voces resultan claramente perjudicados cuando existen altos índices de concentración mediática. Sin embargo, también es cierto que una estructura de propiedad mediática no oligopólica tampoco es suficiente para garantizar la democratización de las comunicaciones. Además, es necesario asegurar la multiplicidad de contenidos en los medios de comunicación, reflejada tanto a nivel político -permitiendo la expresión del conjunto de opiniones políticas-, como cultural -asegurando la posibilidad de que las diferentes culturas nacionales encuentren un canal de expresión.

\subsection{Una aproximación conceptual al campo académico}

¿Cómo estudiar los múltiples resultados de una política pública de medios como la LSCA en el ámbito académico? En principio, el enfoque teórico de este estudio está basado en una sociología de la cultura. Esta perspectiva centra su interés en todos los sistemas significantes, pero, sobre todo, en las prácticas culturales manifiestas. Dicho enfoque requiere el análisis social de instituciones específicamente culturales y la investigación de las relaciones existentes entre éstas y, por un lado, los medios materiales de producción cultural, y, por otro, las formas culturales propiamente dichas (Williams, 1994). De esta manera, abordar la LSCA como objeto de estudio del campo académico implica, necesariamente, el análisis de las relaciones variables entre los productores culturales y las instituciones sociales identificables que los contienen.

¿Cómo podemos conceptualizar esta relación entre los productores culturales y las instituciones de las cuales éstos forman parte? En este caso, debemos comprender la noción de campo académico en el sentido postulado por Bourdieu $(1988,2000)$. Para el autor, los campos son estructuradores y reproductores de la vida social en las sociedades modernas a modo de espacios estructurados en los que hay reglas de juego y objetos por los que se juega. Esto significa que en cada campo hay intereses específicos que son irreductibles a los objetos e intereses propios de otros campos, y que sólo son percibidos por quienes están dotados del 
habitus correspondiente o cultura (de una época, de una clase o de un grupo) en tanto que interiorizada («incorporada») por el individuo bajo la forma de «disposiciones duraderas» que constituyen el principio de su acción (Bourdieu, 1988: 22). De manera que, al considerar los campos científicos como campos de producción simbólica, no pueden entenderse sus productos (conocimiento) sin tomar en cuenta a los agentes productores (investigadores) y las condiciones sociales (institucionalizadas) de su producción (Bourdieu, 2000). Tal es así que la «teoría de los campos» concibe, entonces, a la actividad científica como generadora de productos simbólicos cuyo sentido compartido es la clave primordial de su propia trasformación, y la caracteriza como un proceso sociocultural e ideológico fundado en la trama de las relaciones sociales (Bourdieu, 1988), es decir, como una red de prácticas de comunicación.

¿En qué instituciones se materializa el campo? De acuerdo a Fuentes Navarro (1997:47-48), el campo académico de la comunicación puede ser entendido como el conjunto de instituciones de educación superior destinadas al estudio y a la enseñanza de la comunicación, donde se produce la teoría, la investigación y la formación universitaria. Siguiendo los estudios de Vassallo (Vassallo, 2001: 43-44), el campo académico de la comunicación implicaría la identificación necesaria de tres subcampos:

1- el subcampo científico, implicado en prácticas de producción de conocimiento donde la investigación académica tiene la finalidad de producir conocimiento teórico aplicado por medio de la construcción de objetos, teorías y metodologías;

2- el subcampo educativo, que se define por prácticas de reproducción de ese conocimiento; es decir, la enseñanza universitaria; $y$,

3- el subcampo profesional, caracterizado por prácticas de aplicación del conocimiento y que promueve vínculos de diversos tipos con el mercado laboral.

De este modo, abordar la LSCA como objeto de estudio de la academia, implica necesariamente, examinar el campo a partir no sólo de los textos y contextos del discurso científico, sino abordar, además, tanto su estructura como la práctica científica, a modo de un proceso histórico de conocimiento y reconocimiento de sus agentes e instituciones. 


\subsection{Las producciones científicas: acerca del material de análisis}

Luego de aclarar el contexto y las reglas de producción del conocimiento científico pasemos a definir las producciones académicas mismas. El corpus de análisis del presente trabajo está compuesto por tres fuentes: los artículos referidos a la LSCA pertenecientes a las revistas especializadas en comunicación, las ponencias de los congresos anuales de la Red de Carreras de Comunicación Social y Periodismo de Argentina (RedCom) en torno a la normativa $y$, finalmente, el testimonio oral de los referentes de la disciplina acerca de la cuestión. Con el fin de establecer un recorte temporal se delimitó el lapso transcurrido entre la sanción de la normativa y su declaración de constitucionalidad por parte de la Corte Suprema de Justicia (2009-2013).

Ahora bien, estudiar hechos del pasado reciente implica recurrir a una suerte de historia de las ideas que, como afirma Oscar Terán, es una disciplina que trabaja con discursos, conceptos y palabras, esto es, con representaciones. Para ello se vale fundamentalmente de textos, a través de los cuales intenta restituir la visión que los seres humanos de tiempos pasados tenían de su época y de sus problemas (Terán, 2009). Sin embargo, se hace necesario aclarar que:

El análisis de las representaciones no es un tema separado de la historia, sino que las representaciones son parte de la historia, contribuyen a la historia, son elementos activos en los rumbos que toma la historia, en la manera como se distribuyen las fuerzas, en la manera en cómo la gente percibe las situaciones, tanto desde dentro de sus apremiantes realidades como fuera de ellas (Williams, 1989)

En esta perspectiva de trabajo se privilegia el testimonio oral como fuente fundamental para dar cuenta de los procesos que involucran a los protagonistas de la historia reciente 
porque posibilita dar cuenta de las vivencias de los actores respecto a su propia práctica, es decir, obtener una verdad situada acerca del momento en que fue recogida. Tal como afirma François Dosse (2007), el testimonio oral permite que emerja «el momento de subjetivación del instante» que da cuenta de una «dimensión subjetiva de intensa afectividad».

Por otra parte, esta investigación se centra en las producciones académicas, especialmente las revistas ya que, como sostiene Dosse, son uno de los soportes esenciales de la vida intelectual, una estructura elemental de sociabilidad y un observatorio esencial para analizar tanto la evolución de las ideas -en tanto que lugares de fermentación intelectual donde se cruzan dimensiones políticas y posturas teóricas - como la emergencia de relaciones afectivas, esto es, la parte afectiva y emocional de toda vida colectiva (Ibíd.: 51: 55).

Finalmente, nos queda por aclarar el problema de la interpretación de las producciones científicas analizadas. La dificultad estriba en el hecho de que el objeto de análisis de estas producciones, es decir la LSCA, fue y sigue siendo un significante sujeto a una disputa de sentido por imponer una visión legítima al respecto. En este contexto, las producciones académicas analizadas no pueden pensarse por fuera de las múltiples interpretaciones en conflicto al respecto de la normativa. Es por eso que debemos tener en cuenta que:

El texto no existe por fuera de la historia de sus interpretaciones. Entre el lector y el texto se interpone necesariamente toda una malla de interpretaciones previas. Cada texto ha acumulado una historia de efectos e interpretaciones que son parte constitutiva de su significado para nosotros. El texto no es sino la historia del texto, la historia de sus lecturas. No existe una vía regia de acceso directo, objetivo, neutro, al texto original, virgen de interpretaciones. Pretender esa vía equivale a cancelar nuestra propia historicidad, a saltar por encima de la historia (Tarcus, 2007:21).

Este enfoque plantea un evidente problema teórico-metodológico. ¿Cómo es posible acceder al sentido de un texto si éste se encuentra mediado para nosotros por interpretaciones previas? ¿Interpretar consiste en recuperar la intencionalidad del autor original o es un proceso 
activo abierto a diversas significaciones? ¿Interpretar implica delimitar el análisis sólo al texto estudiado o es necesario ponerlo en relación con las condiciones materiales que posibilitaron su producción?

Si admitimos que las significaciones no son arbitrarias, sino que es posible establecer, a través de la investigación, ciertos patrones de significaciones determinados históricamente, debemos aclarar cómo esto es posible. Siguiendo los desarrollos de Pierre Bourdieu podemos afirmar que el estudio de una obra no cobra sentido al menos que se la vincule con dos series. La primera se refiere a las obras con las que entra en relación según el principio de la intertextualidad, es decir, según el principio de que todo texto reenvía a una escritura múltiple, a una red de relaciones o diálogos que lo producen. La segunda serie con la que debe vincularse una obra, explica Bourdieu, es la del espacio social «en el interior del cual se definen sus propiedades simbólicas pertinentes» (Bourdieu, 2008 [1984]: 296). Lo que debemos dar cuenta, entonces, es de la posición de un autor en relación con la estructura objetiva del campo en el que se inscribe (académico, literario, intelectual, etc.) al mismo tiempo que, desde esta instancia de mediación, a su relación funcional con el campo de poder, esto es, con la reproducción del orden social que se disemina a partir de la naturalización de la desigual distribución de las posiciones de poder en cada campo específico, al mismo que tiempo que por la producción, por parte de estos productores especializados de visiones de mundo, de discursos y saberes legitimadores del orden social (Bourdieu, 1999 [1971]).

\subsubsection{Las entrevistas}

Las entrevistas utilizadas son fruto de una labor colectiva llevada a cabo por un grupo de investigadores pertenecientes al Centro de Investigaciones en Problemáticas Sociosimbólicas "Aníbal Ford» enmarcado dentro de la Facultad de Periodismo y Comunicación Social de la Universidad Nacional de La Plata. Son producto de un Grupo de Trabajo denominado "Historia de los Estudios de Comunicación y Cultura en Argentina» cuyo interés es indagar la conformación de dichos estudios en el país desde los años '60 hasta la actualidad 
situándolos en relación entre la historia de los intelectuales y los contextos y coyunturas que marcaron la constitución del campo.

Desde esta perspectiva se comenzó una serie de entrevistas semiestructuradas a los principales referentes del campo de la comunicación, realizadas entre julio y diciembre del 2013, en las universidades de La Plata, Buenos Aires, Quilmes, Salta, Córdoba y Rosario. Finalmente, se lograron obtener los testimonios de Florencia Saintout, Héctor Schmucler, Pablo Alabarces, Guillermo Mastrini, Martín Becerra, Jorge Huergo, Alejandro Kaufman, Sergio Caletti, Alicia Entel, Alfredo Alfonso, Carlos Mangone, Sergio Caggiano, Vanina Papalini, Víctor Arancibia, Nancy Díaz Larrañaga, Sandra Valdettaro, Alejandra Cebrelli y Diego De Charras.

\subsubsection{Los artículos académicos}

Dentro del amplio universo de revistas especializadas en comunicación, el recorte utilizado para seleccionar las publicaciones que conforman el objeto de análisis de este estudio, se basó en tres parámetros generales: 1- Que las publicaciones pertenezcan a instituciones nacionales conocidas y reconocidas por los agentes del campo. 2- Que los artículos publicados queden enmarcados dentro del lapso temporal delimitado (2009-2013). 3Que una parte significativa de la producción de los artículos de la revista en el periodo de estudio se concentrara en torno a la reflexión de las cuestiones relativas a la LSCA.

Luego de realizar una exhaustiva búsqueda a nivel nacional se llegó a la selección de las siguientes publicaciones:

\begin{tabular}{|l|l|}
\hline \multicolumn{1}{|c|}{ Título de la publicación } & Pertenencia institucional \\
\hline Avatares de la Comunicación y la Cultura (6) & \\
\cline { 1 - 1 } Zigurat (7) & \\
\hline
\end{tabular}




\begin{tabular}{|l|l|}
\hline El Cactus (8) & Universidad Nacional de Córdoba \\
\cline { 1 - 1 } Anuario de Investigaciones (9) & \\
\cline { 1 - 1 } Oficios terrestres (10) & \\
\cline { 1 - 1 } Question (11) & \\
\cline { 1 - 1 } Trampas de la Comunicación y la Cultura (12) & \\
\cline { 1 - 1 } La Ley de la Comunicación Democrática (13) & Universidad Nacional de Quilmes \\
\cline { 1 - 1 } La Trama de la Comunicación (14) & Universidad Nacional de Rosario \\
\hline
\end{tabular}

\subsubsection{Las ponencias}

Las ponencias analizadas en el presente trabajo corresponden a los Congresos Anuales de la Red de Carreras de Comunicación de la Argentina (RedCom)(15) organizados entre 2009 y 2013. Debido a que la Red es de carácter interinstitucional y se extiende por todo el territorio del país, consideramos que el análisis de sus producciones científicas permite brindarnos una excelente muestra del modo en que el campo académico abordó la LSCA como objeto de estudio a nivel nacional. A continuación, pasaremos a detallar aquellos congresos que forman parte de nuestro recorte:

\begin{tabular}{|c|c|c|c|}
\hline Título de Congreso & \multicolumn{2}{|c|}{ Año de } & Sede \\
& realización & \\
\hline XI Congreso REDCOM «Cultura & 22,23 y & Universidad Nacional de \\
de masas y nuevos procesos de & 24 de octubre & Tucumán. Facultad de Filosofía y \\
\hline
\end{tabular}




\begin{tabular}{|c|c|c|}
\hline comunicación» & del 2009 & Letras \\
\hline $\begin{array}{l}\text { XII Congreso REDCOM «Los } \\
\text { desafíos de la comunicación social y el } \\
\text { periodismo en el bicentenario» }\end{array}$ & $\begin{array}{l}7,8 \text { y } 9 \\
\text { de octubre de } \\
2010\end{array}$ & $\begin{array}{l}\text { Facultad de } \text { Ciencias } \\
\text { Políticas y Sociales Universidad } \\
\text { Nacional de Cuyo }\end{array}$ \\
\hline $\begin{array}{l}\text { XIII Congreso REDCOM «Praxis, } \\
\text { fronteras y multiculturalidad: la } \\
\text { comunicación en disputa» }\end{array}$ & $\begin{array}{l}\text { 11,12 y } \\
13 \text { de agosto del } \\
2011\end{array}$ & $\begin{array}{ccc}\text { Sede } & \text { Regional } & \text { Tartagal } \\
\text { (UNSa). Salta } & & \end{array}$ \\
\hline 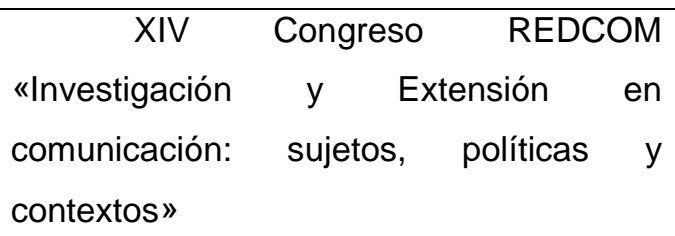 & $\begin{array}{l}28,29 \text { y } \\
30 \text { de junio del } \\
2012\end{array}$ & $\begin{array}{l}\text { Universidad Nacional de } \\
\text { Quilmes }\end{array}$ \\
\hline $\begin{array}{l}\text { XV Congreso REDCOM «Mapas } \\
\text { comunicacionales y territorios de la } \\
\text { experiencia» }\end{array}$ & $\begin{array}{l}\text { 15, } 16 \mathrm{Y} \\
17 \text { de agosto del } \\
2013\end{array}$ & $\begin{array}{l}\text { Facultad de Humanidades } \\
\text { y Ciencias Sociales. Universidad } \\
\text { Nacional de Jujuy. }\end{array}$ \\
\hline
\end{tabular}

\subsection{Métodos y técnicas de recolección de datos}

Para llevar a cabo el trabajo propuesto, se emplearon dos técnicas metodológicas de recolección de datos (a y b). Posteriormente, con el fin de analizar, codificar y sistematizar la información obtenida, se utilizó la denominada Metodología de Identificación de las Características Estructurales del Conocimiento Comunicativo (MICECC) (León Duarte, 2006) (c).

a) La primera técnica de obtención de información es el análisis documental, que responde a tres necesidades. En primer lugar, conocer lo que otros pares científicos han hecho o están realizando en un campo específico; en segundo lugar, conocer segmentos 
específicos de información de algún documento en particular; y por último, conocer la totalidad de información relevante que exista sobre un tema específico (Vickery, 1970: 154). Con esta técnica se analizó las publicaciones institucionales y producciones científicas referentes al caso en cuestión en el periodo seleccionado.

El análisis documental constituye un proceso ideado por el individuo como medio para organizar y representar el conocimiento registrado en los documentos, cuyo índice de producción excede sus posibilidades de lectura y captura. La acción de este proceso se centra en el análisis y síntesis de los datos plasmados en dichos soportes mediante la aplicación de lineamientos o normativas de tipo lingüístico; a través de las cuales se extrae el contenido sustantivo que puede corresponder a un término concreto o a conjuntos de ellos tomados aisladamente, o reunidos en construcciones discursivas. Por consiguiente, su finalidad es facilitar la aproximación cognitiva del sujeto al contenido de las fuentes de información (Perelló, 1998).

b) La otra herramienta para la recolección de datos es la entrevista semiestructurada. En este tipo de entrevista cualitativa el objetivo último del investigador es conocer la perspectiva del sujeto estudiado y comprender sus interpretaciones, percepciones, sentimientos y motivos de sus actos. Podemos definir la entrevista cualitativa como una conversación: a) provocada por el entrevistador; b) realizada a sujetos seleccionados a partir de un plan de investigación; c) en un número considerable; d) que tiene una finalidad de tipo cognitivo; e) guiada por el entrevistador; y) con un esquema de preguntas flexible y no estandarizado (Corbetta, 2007).

Con esta técnica se realizaron una serie de entrevistas a investigadores $y / 0$ docentes de comunicación, así como a los directores de las carreras en el periodo estudiado. La ventaja de esta herramienta metodológica consiste en la flexibilidad de las preguntas lo que concede amplia libertad tanto al entrevistado como al entrevistador, y garantiza al mismo la discusión de todos los temas relevantes y la recopilación de toda la información necesaria. El guión de la entrevista establece un perímetro dentro del cual el entrevistador decide no sólo el orden y la formulación de las preguntas, sino también si se va a profundizar en algún tema. El entrevistador tiene la libertad para desarrollar temas que vayan surgiendo en el curso de la entrevista y que considere importantes para comprender al sujeto entrevistado, aunque no las incluya en el resto de las entrevistas. 
c) Por último, una vez finalizado el trabajo de campo, la información recolectada fue sometida a un análisis cualitativo con el fin de sistematizar los datos obtenidos. Luego de una profunda lectura de la totalidad del material, se procedió a categorizar y codificar los datos en ejes temáticos clasificados y jerarquizados por orden de importancia en función del objeto de estudio. Finalmente, todas las categorías generadas fueron volcadas en una matriz con el objetivo de comparar la información de diferentes fuentes acerca de una misma cuestión.

Sin embargo, es necesario aclarar que el análisis es un proceso cíclico y una actividad reflexiva; el proceso analítico debe ser amplio y sistemático, pero no rígido, los datos se fragmentan y dividen en unidades significativas, pero se mantiene una conexión con el total; y los datos se organizan según un sistema derivado de ellos mismos. Como un todo, el análisis es una actividad inductiva guiada por los datos (Coffey y Atkinson, 2003: 12). Respecto de la codificación, esta definición indica que las categorías que se elaboran deben ajustarse a los datos y no a la inversa, de manera que no se utilizan conceptos de forma estática y definitiva que obliguen a los datos a «encajar» en ellos.

\subsection{Acerca de la propuesta metodológica de análisis}

Como se mencionó anteriormente, con el fin de identificar, analizar, codificar y sistematizar la información obtenida de las producciones científicas relativas a la LSCA, se utilizó la denominada Metodología de Identificación de las Características Estructurales del Conocimiento Comunicativo (MICECC) (León Duarte, 2006). Esta propuesta metodológica aspira a un análisis que no se limite sólo al texto estudiado, sino que, además, requiere poner a dicho texto en relación con las condiciones materiales que posibilitaron su producción. Precisamente por eso, además de analizar el contenido de las producciones científicas en sí, prestamos especial atención a los siguientes indicadores relativos a su contexto de producción: 


\begin{tabular}{|c|cc|}
\hline \multicolumn{2}{|c|}{ Indicadores de análisis } \\
\hline & $\bullet$ & Quién es el autor del texto \\
& $\bullet$ & A quién(es) se dirige \\
& $\bullet$ & Quiénes son los protagonistas del texto y qué se dice de ellos \\
& $\bullet$ & A qué realidad y contexto se dirige. \\
\hline & $\bullet$ & Cuál es el lugar del autor en relación con la posición que ocupa \\
\hline & & Cuál es la afirmación ético-política de su propuesta \\
\hline & & Cuáles son los procedimientos de exclusión del discurso \\
\hline
\end{tabular}

Para desarrollar estas categorías analíticas se recurrió a varias propuestas teóricometodológicas. Por un lado, para dar cuenta de la posición que ocupa determinado autor dentro del campo académico, así como la afirmación ético-política de su propuesta nos basamos en las conceptualizaciones del Pierre Bourdieu acerca del campo científico. De acuerdo al autor, (1983:11-35; 2000:11-142), para dar cuenta desde dónde se habla es necesario identificar el punto de vista que refleja las distintas posiciones y perspectivas del campo intelectual y político. De esta manera, es posible identificar lo que se produce al interior del texto como parte de un principio generador y unificador del conjunto de prácticas epistemológicas e ideológicas de un determinado grupo académico. Los desarrollos teóricos de Bourdieu, permitieron, además, no sólo rescatar la evidencia de un determinado posicionamiento en el texto, sino que, a partir de él, definir la posición del autor dentro del sistema de relaciones que se dan en el campo académico.

Para realizar esta tarea, seguimos los tres principios metodológicos propuestos por Bourdieu (1983:20-35). Estos preceptos fueron utilizados para codificar la categoría 2 de nuestros indicadores de análisis (ver cuadro anterior). Si bien el análisis del funcionamiento del campo académico se desarrollará posteriormente, nos limitaremos a enunciar a continuación cuáles fueron los aspectos que puntualizamos para dar cuenta de la posición de los agentes dentro del campo siguiendo a Bourdieu: 
1-Un análisis de la producción y la posición de los investigadores en la estructura del campo o con respecto a él.

2-Un análisis de las relaciones objetivas y las lógicas específicas que los propios grupos en competencia realizan por la obtención de la legitimidad intelectual, en un momento dado, en la estructura del campo intelectual.

3- Un análisis del sistema que dé cuenta del principio que logra unificar y generar todas las prácticas, es decir, el habitus de la práctica científica que se analiza y que es característica de un determinado grupo científico.

Por otra parte, para desarrollar la categoría de los mecanismos de exclusión de discurso en las producciones científicas, se recurrió a la propuesta metodológica de Foucault (1978:7-21; 1983:9-64; 1990a:7-49; 1990:3-355). Para el autor, la producción de un discurso, entendido como la materialidad de una cosa pronunciada o escrita, supone siempre un procedimiento de exclusión. Esto significa que una producción discursiva implica una serie de mecanismos de control y selección que tienen por función dominar el hecho aleatorio e imponerle un sentido. Si bien el autor clasifica estos mecanismos en externos e internos en relación a un discurso dado, para el presente análisis nos interesan solamente los segundos y, dentro de ellos, las categorías de «comentario» y «organización de las disciplinas».

En efecto, el comentario tiene por función «decir por primera vez aquello que, sin embargo, había sido ya dicho" (Foucault, 1974: 29). Este mecanismo permite construir indefinidamente nuevos discursos y, por lo general, se aplica a textos religiosos, literarios y jurídicos. Su importancia radica en que lo que se diga de un discurso, siempre afectará su sentido a través de la interpretación que se hace a posteriori de él. En nuestro caso, dado que las producciones científicas son realizadas acerca de una norma jurídica significada y resignificada, este procedimiento de exclusión se presenta como preponderante para el análisis. 
Por otra parte, la organización de las disciplinas es aquella que permite reactualizar constantemente las reglas del discurso. En efecto, la disciplina posibilita la construcción de nuevos enunciados brindando un sistema anónimo y ajeno al productor de un discurso dado. Este conjunto de reglas funciona como un mecanismo de exclusión ya que para conformar una disciplina es necesario orientar la dirección discursiva hacia determinado conjunto de objetos y poder inscribirse en cierto tipo de horizonte teórico. De esta forma, pertenecer a un determinado campo de saber cómo el de la comunicación condiciona la manera en que sus agentes se aproximan a determinado objeto de estudio, en este caso, la LSCA.

Ahora bien, con el fin de revertir los mecanismos de control del discurso en las producciones científicas analizadas, utilizaremos los procedimientos sugeridos por Foucault:

\begin{tabular}{|c|c|}
\hline Trastrocamiento & $\begin{array}{l}\text { Esto implica la necesidad de reconocer en esas figuras que se consideran } \\
\text { la fuente de los discursos el juego negativo de un corte y un enrarecimiento } \\
\text { del discurso. }\end{array}$ \\
\hline Discontinuidad & $\begin{array}{l}\text { Los discursos deben ser tratados como prácticas discontinuas que se } \\
\text { cruzan, a veces se yuxtaponen, pero que también pueden ignorarse y } \\
\text { excluirse. }\end{array}$ \\
\hline Especificidad & $\begin{array}{l}\text { No abordar el discurso como un conjunto de significaciones previas sino } \\
\text { concebirlo como una práctica que es impuesta por nosotros. }\end{array}$ \\
\hline Exterioridad & $\begin{array}{l}\text { No ir del discurso hacia su núcleo interior y oculto intentando desentrañar } \\
\text { una significación que se manifestarían en él sino, por el contrario -a partir del } \\
\text { discurso mismo-, de su aparición y de su regularidad, ir hacia sus } \\
\text { condiciones externas de posibilidad, hacia lo que da motivo a la serie } \\
\text { aleatoria de esos acontecimientos y que fija los límites. }\end{array}$ \\
\hline
\end{tabular}




\section{Referencias bibliográficas}

Anderson, P. (1999). Más allá del neoliberalismo: Lecciones para la izquierda, en Sader, E. y Gentilli, P. (comps.), La trama del neoliberalismo. Mercado, crisis y exclusión social, Buenos Aires, CLACSO-EUDEBA.

Borón, A. (2008). Teoría(s) de la dependencia, Conferencia del ciclo del mismo nombre organizada por la Facultad de Ciencias Económicas de la Universidad de Buenos Aires y pronunciada el 30 de mayo de 2008.

Becerra, M. (2012). Nuevo siglo, nuevas reglas de juego. Revista El Cactus N. ํ 1, Córdoba, U.N.C.

Becerra, M. (2009). Texto y contexto. Revista Tram(p)as de la Comunicación y la Cultura N.ำ 67, Buenos Aires, Fac. de Periodismo y Comunicación Social - U.N.L.P.

Bourdieu, P. (1976). El campo científico, en Intelectuales, política y poder. Buenos Aires. EUDEBA.

Bourdieu, P. (2000). Los usos sociales de la ciencia, Buenos Aires, Nueva Visión

Bourdieu, P. (1983). Campo de poder y campo intelectual, Buenos Aires.

Bulla, G. (2013). Un balance provisorio. A más de tres años de la sanción de la ley N.ํㅜ 26.522. Revista Zigurat N. ำ 7, Buenos Aires, Fac. de Ciencias Sociales - U.B.A.

Busso, N. y JAIMES, D. (2011). La cocina de la ley. El proceso de incidencia en la elaboración de la Ley de Servicios de Comunicación Audiovisual en Argentina. Buenos Aires, FARCO.

Bustamante, E. (2004). Políticas de comunicación y cultura: nuevas necesidades estratégicas. Questiones Publicitarias, 1 (9), pp. 9-31.

Califano, B. (2007). Medios y políticas de comunicación en Argentina bajo el gobierno de Néstor Kirchner (2003 - 2007). Buenos Aires. Fac. de Ciencias de la Comunicación - U.B.A. 
Coffey, A. y Atkinson, P., (2003). Capítulo 1: Variedad de datos y variedad de análisis y Capítulo 2: Los conceptos y la codificación. En Encontrar el sentido a los datos cualitativos, Colombia: Universidad Nacional de Antioquia.

Corbetta P. (2007). Metodología y Técnicas de investigación social. McGraw Hill / Interamericana De España, S.a.

Díaz, E. (1997). Conocimiento, ciencia y epistemología. En: Metodología de las ciencias sociales. Buenos Aires, Biblos.

Dosse, F. (2007), La marcha de las ideas. Historia de los intelectuales. Historia intelectual. Publicaciones de la Universidad de Valencia.

Foucault, M. (1988). Las palabras y las cosas. Una arqueología de las ciencias humanas. México. Siglo XXI.

Foucault, M. (1990). La arqueología del saber. México. Siglo XXI.

Foucault, M. (1974). El orden del discurso, Barcelona, Tusquets.

Fuentes Navarro, R. (1992). Un campo cargado de futuro. El estudio de la comunicación en América Latina. México, FELAFACS.

Fuentes Navarro, R. y Vidales Gonzáles, C. (2010) Fundaciones y fundamentos del estudio de la comunicación. CEC y TE NL.

Fuentes-Navarro, R. (Coord.). (2004). Producción, circulación y reproducción académicas en el campo de la comunicación en México". Tlaquepaque, Jalisco: ITESO.

Gómez Germano, G. (2009). Ley de Servicios de Comunicación Audiovisual de la Argentina. Revista Tram(p)as de la Comunicación y la Cultura N. 67, Buenos Aires, Fac. de Periodismo y Comunicación Social - U.N.L.P.

González Almandoz, D. (2013). La comunicación como derecho: políticas estatales, prácticas de sujetos y ampliaciones democráticas. Revista Question N. ํ 38, Buenos Aires, Fac. de Periodismo y Comunicación Social - U.N.L.P. 
Graziano, M. (1998). Política o ley: debate sobre el debate. Revista Espacios, Buenos Aires, Fac. de Filosofía y Letras - U.B.A.

Guzmán, V. (2012). Ciudadanía comunicativa en Argentina. Los foros participativos de consulta pública sobre la propuesta de proyecto de Ley de Servicios de Comunicación Audiovisual". Revista Question N. ํ 33, Buenos Aires, Fac. de Periodismo y Comunicación Social - U.N.L.P.

Tarcus, H. (2007), Marx en la Argentina. Sus primeros lectores obreros, intelectuales y científicos (1871-1910). Buenos Aires, Siglo XXI.

Jensen, C. (2009). Reflexiones sobre el anteproyecto. Revista Tram(p)as de la Comunicación y la Cultura N.․67, Buenos Aires, Fac. de Periodismo y Comunicación Social - U.N.L.P.

Jessop, B. (1982). Hacia una perspectiva teórica del estado. The capitalist state. Marxist theories and methods, New York University Press.

Kauffer, E. (2002). Las políticas públicas: algunos apuntes generales. Ecofronteras, 16, pp. 2-5.

Kriger, C (2009). Cine y peronismo. El estado en escena. Buenos Aires: Siglo XXI.

Laclau, E. y Mouffe, C. (2004) Hegemonía y estrategia socialista, Fondo de Cultura Económica, Argentina.

León Duarte, G. (2006) Sobre la institucionalización del campo académico de la comunicación en América Latina. Una aproximación a las características estructurales de la investigación latinoamericana en comunicación. Tesis doctoral publicada por la Universidad Autónoma de Barcelona.

Ley 26.522 de Servicios de Comunicación Audiovisual.

Loreti, D. (2013). El caso argentino. Acerca de la nueva Ley de Servicios de Comunicación Audiovisual № 26.522. Revista Zigurat N. ำ , Buenos Aires, Fac. de Ciencias Sociales - U.B.A. 
Loreti, D. (2014). La actualización de un debate pendiente. Apuntes sobre el fallo de la Corte Suprema que declaró la constitucionalidad de la Ley de Servicios de Comunicación Audiovisual. En Loreti D. y Sabsay D., El fallo grupo clarín: dos puntos de vista, Buenos Aires.

Lozano, M. (2010). Un compromiso sostenido, en Ottaviano, C. y Lozano, M. (comps.) La ley de la comunicación democrática, Buenos Aires, U.N.Q.

Mastrini, G. y Becerra, M. (2010). Periodistas y Magnates. Estructura y concentración de las industrias culturales en América Latina. Buenos Aires, Prometeo.

O’Donnell, G. (2010). Democracia, agencia y estado. Teoría con intención comparativa. Buenos Aires: Prometeo.

Terán, O. (2012). Historia de las ideas en la Argentina. Siglo XXI.

Oszlak, O. y O'Donnell, G. (1984). Estado y políticas estatales en América Latina: hacia una estrategia de investigación. En G. Flores y J. Nef (comps.), Administración pública. Perspectivas críticas. San José de Costa Rica: ICAP.

Ottaviano, C (2010). Nadie reclama un derecho que no conoce. En Ottaviano, C. y Lozano, M. (comps.) La ley de la comunicación democrática, Buenos Aires, U.N.Q.

Perelló, J. (1998), Sistemas de indización aplicados en bibliotecas: clasificaciones, tesauros y encabezamientos de materias. En: Magán Wals, J. ed. Tratado básico de biblioteconomía. 3a. ed. Madrid: Complutense. p. 200-203.

Roncagliolo, R. (1983). Comunicación y democracia en el debate internacional. Revista Chasqui, № 7 , julio/diciembre.

McBride, S. (1980). Un solo Mundo. Voces Múltiples. Comunicación e Información en Nuestro Tiempo, Sean, Fondo de Cultura Económica (FCE) y UNESCO, México.

Vassallo, I. (2001). El campo de la comunicación: reflexiones sobre su estatuto disciplinar. En Comunicación: campo y objeto de estudio. Perspectivas reflexivas latinoamericanas, Guadalajara, ITESO/UC/UAA/U. de G; pp. 43-58. 
Vickery, B. (1970), Techniques of information retrieval. Londres: Butterworths.

Williams, R. (1989). La política del modernismo. Contra los nuevos conformistas. Manantial.

Williams, R. (2000). Marxismo y literatura. Ediciones Península. Barcelona.

Williams, R. (1994). Sociología de la cultura. Barcelona. Paidós.

\section{Notas}

(1)Para una reconstrucción histórica del proceso de sanción de la Ley de Servicios de Comunicación Audiovisual véase el Capítulo II.

(2) Una obra emblemática de ese período fue Comunicación y cultura de masas, publicada por Antonio Pasquali en 1964. Allí el autor sentó las bases para entender a la comunicación como un problema de poder económico antes que de un análisis inmanente de los mensajes (Graziano, 1997). Otra publicación en el mismo sentido fue Neocapitalismo y comunicación de masas, escrita por Heriberto Muraro, en 1974.

(3) El Informe planteó la necesidad de considerar diversos aspectos (Mac Bride, 1980)

1- Que los países amplíen las fuentes de información que necesitan los ciudadanos en su vida cotidiana.

2- Se debe abolir la censura o el control arbitrario de la información.

3- Los obstáculos y las restricciones que se derivan de la concentración de la propiedad de los órganos de información merecen analizarse para encontrar sus vías democratizadoras.

4- Tomar medidas jurídicas eficaces para limitar la concentración y monopolización; conseguir que las empresas trasnacionales acaten los criterios y las condiciones específicas definidas en la legislación y en las políticas de desarrollo nacional; invertir la tendencia a la reducción del número de responsables cuándo está aumentando la eficacia de la comunicación y la dimensión del público; y reducir la influencia de la publicidad sobre la política de redacción y los programas de radiodifusión. 
(4) Las leyes de "Reforma del Estado" y "Emergencia Económica" que se sancionaron bajo la presidencia de Carlos Menem permitieron privatizar o entregar en concesión numerosas empresas y activos estatales, transformando radicalmente el papel del Estado en la economía nacional.

(5)A modo de ejemplo, tomemos el caso emblemático del Grupo Clarín previo a la sanción de la Ley de Servicios de Comunicación Audiovisual. Entre sus principales medios figuraban: el diario Clarín, el diario deportivo Olé, el Canal 13 de televisión abierta, la empresa Cablevisión de televisión de pago, varias señales de cable, las radios Mitre y FM 100, la empresa Prima S.A. proveedora de servicios de Internet y productora de contenidos digitales, la participación accionaria en diversos medios gráficos en el interior a través de la compañía inversora CIMECO S.A. (junto con S.A. La Nación) y la editorial Tinta fresca. Asimismo, es socio de la empresa Papel Prensa S.A., posee imprentas propias, es propietario de la productora de contenidos Artear S.A., está asociado con otras productoras como Pol-Ka y Patagonik, y participa de la agencia de noticias DyN.

(6) http://ppct.caicyt.gov.ar/index.php/avatares

(7) http://comunicacion.sociales.uba.ar/?page id=1092

(8) http://www.eci.unc.edu.ar/comunicacion/elcactus

(9)http://perio.unlp.edu.ar/ojs/index.php/anuario

(10) http://www.perio.unlp.edu.ar/oficios/index.html

(11) http://perio.unlp.edu.ar/ojs/index.php/question/

(12)http://www.revistatrampas.com.ar/

(13) http://www.defensadelpublico.gob.ar/es/ley-comunicacion-democratica-0 Publicación de la Defensoría del Público en articulación con la Universidad Nacional de Quilmes (UNQ). - Más información en http://www.defensadelpublico.gob.ar/es/ley-comunicacion-democratica0\#sthash.Irew9JII.dpuf

(14) http://www.latrama.fcpolit.unr.edu.ar/index.php/trama

(15) De acuerdo al sitio web de REDCOM la institución fue creada en el año 1998 y está integrada por diferentes universidades de todo el país y entre sus objetivos principales se propone:

"Crear una red interinstitucional de carreras, departamentos, escuelas, facultades y/o profesores e investigadores que enseñen cursos de grado y/o postgrado universitario en las 
Question/Cuestión, Vol. 3, Nº70

Diciembre 2021

ISSN 1669-6581

carreras de Periodismo y/o Comunicación Social, a los fines de promover la mejora de la calidad de la enseñanza en el propio campo". 